\title{
Corrigendum: Thymoquinone Inhibits Virulence Related Traits of Cronobacter sakazakii ATCC 29544 and Has Anti-biofilm Formation Potential
}

\author{
Chao Shi ${ }^{1}$, Chunhong Yan ${ }^{2,3}$, Yue Sui ${ }^{1}$, Yi Sun ${ }^{1}$, Du Guo ${ }^{1}$, Yifei Chen ${ }^{1}$, Tong Jin ${ }^{1}$, \\ Xiaoli Peng ${ }^{1}$, Linlin $\mathrm{Ma}^{4}$ and Xiaodong $\mathrm{Xia}^{1 *}$ \\ ${ }^{1}$ College of Food Science and Engineering, Northwest A\&F University, Yangling, China, ${ }^{2}$ College of Animal Science and \\ Technology, Northwest A\&F University, Yangling, China, ${ }^{3}$ School of Life Science and Technology, Xi'an Jiaotong University, \\ Xi'an, China, ${ }^{4}$ Xi'An Yurun Agricultural Products Global Sourcing Co., LTD., Xi'an, China
}

Keywords: Cronobacter sakazakii, Thymoquinone, sub-inhibitory concentration, HT-29 cells, Virulence Factors

\section{A corrigendum on}

Thymoquinone Inhibits Virulence Related Traits of Cronobacter sakazakii ATCC 29544 and Has Anti-biofilm Formation Potential

by Shi, C., Yan, C., Sui, Y., Sun, Y., Guo, D., Chen, Y., et al. (2017). Front. Microbiol. 8:2220. doi: 10.3389/fmicb.2017.02220

\section{OPEN ACCESS}

Edited and reviewed by: Lanming Chen,

Shanghai Ocean University, China

*Correspondence: Xiaodong Xia foodscixiaodong@yahoo.com

Specialty section

This article was submitted to Food Microbiology, a section of the journal

Frontiers in Microbiology

Received: 05 January 2018 Accepted: 07 February 2018 Published: 20 February 2018

Citation:

Shi C, Yan C, Sui Y, Sun Y, Guo $D$, Chen $Y$, Jin T, Peng $X, M a L$ and $X i a X$ (2018) Corrigendum: Thymoquinone Inhibits Virulence Related Traits of

Cronobacter sakazakii ATCC 29544 and Has Anti-biofilm Formation

Potential. Front. Microbiol. 9:290. doi: 10.3389/fmicb.2018.00290
In the first paragraph in the "Introduction" of this article, the number of Cronobacter species was incorrectly described as ten. Actually, Cronobacter pulveris, Cronobacter helveticus and Cronobacter turicensis were removed from the genus in 2014.

The original sentence should be corrected as follows: Cronobacter spp. is currently considered to consist of 7 species: Cronobacter sakazakii, C. malonaticus, C. universalis, C. dublinensis, C. muytjensii, C. condiment, and C. zurichensis (Stephan et al., 2014), among which C. sakazaii is one of the two group 1 clinically relevant species that form the majority of the clinical isolates. The authors regret this error.

The original article has been updated.

\section{REFERENCES}

Stephan, R., Grim, C. J., Gopinath, G. R., Mammel, M. K., Sathyamoorthy, V., Trach, L. H., et al. (2014). Re-examination of the taxonomic status of Enterobacter helveticus, Enterobacter pulveris and Enterobacter turicensis as members of the genus Cronobacter and their reclassification in the genera Franconibacter gen. nov. and Siccibacter gen. nov. as Franconibacter helveticus comb. nov., Franconibacter pulveris comb. nov. and Siccibacter turicensis comb. nov., respectively. Int. J. Syst. Evol. Microbiol. 64, 3402-3410. doi: 10.1099/ijs.0.059832-0

Conflict of Interest Statement: The authors declare that the research was conducted in the absence of any commercial or financial relationships that could be construed as a potential conflict of interest.

Copyright () 2018 Shi, Yan, Sui, Sun, Guo, Chen, Jin, Peng, Ma and Xia. This is an open-access article distributed under the terms of the Creative Commons Attribution License (CC BY). The use, distribution or reproduction in other forums is permitted, provided the original author(s) and the copyright owner are credited and that the original publication in this journal is cited, in accordance with accepted academic practice. No use, distribution or reproduction is permitted which does not comply with these terms 